\title{
On-Pump Beating Heart versus Off-Pump Coronary Artery Bypass Graft Surgery: Short- Term Follow-up Outcomes of a Single Center
}

\author{
Ekin İlkeli ${ }^{1}$, Ali Cemal Düzgün ${ }^{2}$ \\ ${ }^{1}$ Department of Cardiovascular Surgery, Düzce State Hospital, Düzce, Turkey \\ ${ }^{2}$ Department of Cardiovascular Surgery, Ankara Training and Research Hospital, Ankara, Turkey
}

\begin{abstract}
Background and Aim: Novel surgical approaches are gaining attention in an attempt to overcome possible adverse events following coronary artery bypass graft (CABG) surgery. This single-center study aimed to evaluate and compare the early postoperative outcomes of onpump beating-heart (OPBH-CABG) versus off-pump CABG surgery in similar risk groups with a total number of 1-2 grafts. Methods: The records of a total of 229 patients who underwent non-emergency, primary, isolated, either OPBH-CABG ( $n=32$ ) or off-pump CABG (n = 197) surgery were retrospectively evaluated. Reported outcome measures included baseline data, the number of coronary artery grafts, prophylactic intra-aortic balloon pump, time of extubation from mechanical ventilation, duration of stay in the coronary ICU and hospital, as well as early postoperative complications, in-hospital and early postoperative mortality. Results: The groups were identical with regards to age and BMI. The patients in the offpump group received a higher number of grafts ( $1.84 \pm 0.36$ vs. $1.18 \pm 0.39, p<0.0001)$. The OPBH-CABG group had a higher Euroscore II score (2.514 \pm 1.68 vs. $1.706 \pm 1.93, \mathrm{p}=0.021)$. The time to extubation after the surgery, postoperative length of ICU stay, and total length of hospital stay were similar between the groups ( $\mathrm{p}=0.2228 ; \mathrm{p}=0.098 ; \mathrm{p}=0.717$, respectively). The incidence of arrythmia and atrial fibrillation was higher in the on-pump group (12.5\% vs. $2.53 \%, \mathrm{p}<0.05)$. One patient in the on-pump, and four patients in the off-pump groups deceased due to postoperative complications. Conclusions: OPBH-CABG surgery is comparable to off-pump CABG in terms of early postoperative outcomes. In patients who underwent $\mathrm{OPBH}-\mathrm{CABG}$, the risk of arrythmia and atrial fibrillation should be of concern and solved with optimal strategies.
\end{abstract}

Keywords: coronary artery bypass graft, on-pump beating-heart, arrhythmia, atrial fibrillation

\section{ARTICLE HISTORY}

Received: January 22, 2021

Accepted: February 28, 2021

\section{CORRESPONDENCE}

Ekin İlkeli

Aziziye Mahallesi, şht. Ramazan Gel

Cd. No: 7

81010 Düzce, Turkey

Tel: +905056384372

E-mail: ekinilkeli@hotmail.com

\section{INTRODUCTION}

Coronary artery bypass grafting (CABG) had been a standard surgical procedure for the improvement of coronary artery disease and related conditions. Owing to the current interventions and developments in the areas of cardiovascular surgery and anesthesiology, the mean age of the patients undergoing CABG has increased in the last decades. ${ }^{1}$ 
In conventional $\mathrm{CABG}$, intraoperative hypothermia, aortic cannulation, and implementation of cross-clamping have been shown to be associated with numerous severe complications including stroke, arrythmia and dysrhyhmias, neurocognitive perturbations, and increased rate of mortality. ${ }^{2-5}$ Hence, novel approaches are of crucial importance for the safety and increased survival of patients in the clinical and age-related high-risk group.

On-pump beating-heart CABG (OPBH-CABG) surgery is a relatively novel hybrid approach to coronary artery grafting, which aims to stabilize hemodynamic parameters during the operative period. This has specific benefits such as reduced preload alongside the afterload, which decreases the oxygen demand of the myocardial tissue. ${ }^{6}$

However, despite reported benefits for the high clinical risk patients and older individuals, the clinical benefits of OPBH-CABG are still under discussion due to reported adverse effects on graft patency, revascularization, and cardiac output. On the other hand, the longer follow-up data of more than 5 years in multicenter trials comparing the efficiency of OPBH-CABG with off-pump CABG presented better outcomes with the former, possibly as a result of improved experience, skill, and patient selection criteria over time. ${ }^{7,8}$

In the present retrospective study, we aimed to report our clinical experience with high-risk patients who underwent OPBH-CABG surgery, and to compare the preoperative, intraoperative, and postoperative outcomes with the data of a cross-sectional group with similar characteristics operated with off-pump CABG surgery.

\section{MATERIALS AND METHODS}

\section{PATIENT SELECTION CRITERIA}

A total of 229 patients who underwent CABG between April 2017 and August 2019 were included in the study. The study was conducted in accordance with the Declaration of Helsinki, and ethical approval was obtained from the institutional review board.

Patients with mechanical defects related to the ventricular septum, papillary muscles, and mitral valve, as well as a concurrent cardiac condition that required intervention in the same session were excluded. Emergency surgery procedures, cases switched to open heart surgery following initiation of OPBH-CABG, patients with chronic renal impairment with a history of regular dialiysis and/or a high serum creatinine level $\geq 2 \mathrm{mg} / \mathrm{dL}$ were also excluded. Since the number of grafts in the OPBH-CABG cases were 1 and 2, conventional CABG cases with more than 2 vascular graftings were further excluded.
Of the remaining, we evaluated the retrospective chart data of 32 OPBH-CABG and 197 off-pump CABG cases.

\section{STUDY VARIABLES}

Demographic variables included common variables such as age, gender, and body mass index. Preexisting comorbidities including diabetes mellitus, hypertension, chronic obstructive pulmonary disease, thyroid disease, cerebrovascular disease, carotid artery disease, New York Heart Association (NYHA) class, European System for Cardiac Operative Risk Evaluation II model (EuroSCORE II), and ASA status were recorded.

Intraoperative variables were the number of coronary artery grafts, additional dosing of systemic heparinization, requirement for support with inotropic, adrenergic, noradrenergic, dopaminergic, calcium sensitiser agents, prophylactic intra-aortic balloon pump, pacemaker requirement, and duration of surgery.

We also recorded the need for postoperative treatment with vasopressor, positive inotropic agents, time of extubation from mechanical ventilation, duration of stay in the coronary ICU and hospital, postoperative complications, in-hospital mortality, mortality within 30 days after the CABG surgery.

\section{SURGICAL PROCEDURES}

Median sternotomy was performed, and the left internal mammarian artery (LIMA) was used for grafting the left anterior descending artery in all cases. The remaining grafting was performed using the graft obtained from the saphenous vein.

OPBH-CABG patients were operated under cardiopulmonary bypass $(\mathrm{CPB})$ on the working heart using a mechanical stabilizer (Abbott, USA), 3-way Swan-Ganz catheter with radial artery cannula, central catheter, and thermodilution catheter. Chest electrodes for ECG and temperature probes were placed. The body temperatures of the patients were kept at $36^{\circ} \mathrm{C}$, and a cell salvage device was used during the operations. A standard anesthesia protocol consisting of fentanyl citrate (Fentanyl, Janssen), midazolam (Dormicum, Roche), and pancuronium (Pavulon, Organon) was applied for sedation and induction of anaesthetia.

\section{OFF-PUMP CABG PROCEDURE}

After the median sternotomy, the LIMA was prepared as a pedicle graft. Following the administation of heparin (3 
$\mathrm{mg} / \mathrm{kg}$ ), an aortic cannula and a right atrial "two-stage" venous cannula were placed when the activated clotting time (ACT) reached over 480 seconds. An oxygenator (Dideco, Italy) and a roller pump (Sarns 7000, USA) were used for CPB. Patients were cooled to $28-30{ }^{\circ} \mathrm{C}$, and antegrade warm blood cardioplegia $(15 \mathrm{~mL} / \mathrm{kg}$ ) was administered following cross-clamping. The pump speed was adjusted to keep arterial blood pressure at $60-90 \mathrm{mmHg}$. Cold blood cardioplegia was given every 20 minutes, and hot blood cardioplegia was given for 5 minutes prior to the cross-clamp being removed. Distal anastomoses were sealed with 7/0 polypropylene suture (Prolene, Ethicon, Somerville, NJ), proximal anastomoses were sutured with 5/0 polypropylene during warm-up with a side clamp. Heparin effect was neutralized with protamine prior to the termination of $\mathrm{CPB}$.

\section{ON-PUMP CABG PROCEDURE}

For the OPBH-CABG procedure, a $1 \mathrm{mg} / \mathrm{kg}$ dose of heparin was administered, and ACT was kept over 300 seconds. The order was determined as anterior ventricular, posterior, and lateral vessels, respectively. A deep pericardial sling suture was placed in the left superior pulmonary vein area in an attempt to reach the right and circumflex coronary artery. During the operation, the operating table was placed in the Trendelenburg position and rotated to the right. A bulldog clamp was placed in the proximal region following arteriotomy in all anastomoses. Distal anastomoses were sealed with the help of a mechanical stabilizer (Octopus II, Medtronic, USA) and 7/0 polypropylene suture. Protamin-induced heparin neutralization was performed at the end of the operation.

Inotropic support was provided for patients with a systolic blood pressure of $<90 \mathrm{mmHg}$ for both groups.

\section{STATISTICAL ANALYSIS}

All statistical analyses were carried out using GraphPad version 8.3 (GraphPad Software, La Jolla, California, USA). Continuous data were presented as mean \pm standard deviation (SD), and categorical variables were expressed as numbers (percentages). Normally and nonnormally distributed continuous variables were compared using Student's t-test and the Mann-Whitney U test, respectively. Fisher's exact test or the chi-square test was used to compare categorical variables. Cumulative survival curves were constructed using the KaplanMeier method. A p value of less than 0.05 was considered statistically significant.

\section{RESULTS}

Two hundred twenty-nine patients with coronary artery disease who underwent CABG surgery of 1 or 2 grafts were included in the study. Among these, 32 patients received OPBH-CABG, and the remaining 197 patients underwent off-pump CABG. The baseline characteristics of the entire cohort are shown in Table 1.

The mean age of the on-pump group was $65.31 \pm 10.16$ years, whereas the off-pump group had a mean age of $62.10 \pm 9.637$ years $(p=0.085)$. The female to male ratio was higher in the off-pump group (1/31 vs. 37/160, p <0.05). The BMI of the patients did not differ between the groups $\left(25.99 \pm 3.45 \mathrm{~kg} / \mathrm{m}^{2}\right.$ vs. $\left.26.70 \pm 3.82 \mathrm{~kg} / \mathrm{m}^{2}, \mathrm{p}=0.14\right)$.

Patients in the on-pump group had a higher prevalence of diabetes and thyroid disease compared to the off-pump group ( $43.7 \%$ vs. $27.4 \%, \mathrm{p}<0.05 ; 12.5 \%$ vs. $4.5 \%$, p $<0.05$, respectively).

Although the ratios of patients with congestive heart failure, hypertension, chronic renal disease, cerebrovascular disease, and carotid artery disease were higher in the on-pump group, the statistical analysis did not yield a significant difference.

Two patients in the on-pump group, and four patients in the off-pump group received prophylactic preoperative intra-aortic balloon pump (IABP) support $(\mathrm{p}=0.66)$.

There was a statistically signifcant difference in the number of grafts between groups, with a higher number of grafts in the off-pump group $(1.84 \pm 0.36$ vs. $1.18 \pm 0.39$, $\mathrm{p}<0.0001$ ).

A higher Euroscore II score was observed in the onpump group $(2.514 \pm 1.68$ vs. $1.706 \pm 1.93, p=0.021)$.

Early postoperative variables and outcomes are presented in Table 2. The time to extubation after the surgery was similar between the on-pump and the off-pump groups ( $20.94 \pm 30.63$ hours vs. $12.08 \pm 12.01$ hours, $p=$ 0.2228 ). The postoperative length of ICU stay was $1.58 \pm$ 1.47 days in the on-pump group and $1.21 \pm 0.84$ days in the off-pump group ( $\mathrm{p}=0.098$ ).

The total length of hospital stay was $10.97 \pm 4.25$ days for the on-pump group and $11.13 \pm 4.10$ days in the offpump groups ( $\mathrm{p}=0.717$ ).

There were no significant differences between the groups with regards to pulmonary, gastrointestinal, neurological, and local complications. The incidence of arrythmia and atrial fibrillation was higher in the on-pump group ( $12.5 \%$ vs. $2.53 \%, \mathrm{p}<0.05)$.

One patient in the on-pump, and three patients in the off-pump surgery groups deceased due to postoperative pulmonary complications. One patient in the off-pump 
TABLE 1. Baseline characteristics and operation data of the entire patients

\begin{tabular}{lccc}
\hline & $\begin{array}{c}\text { On-pump } \\
\text { beating-heart } \\
\text { surgery } \\
\text { (n= 32) }\end{array}$ & $\begin{array}{c}\text { Off-pump surgery } \\
(\mathbf{n}=\mathbf{1 9 7})\end{array}$ & p value \\
& $65.31 \pm 10.16$ & $62.10 \pm 9.637$ & 0.085 \\
\hline Age, years & $1 / 31$ & $37 / 160$ & $<0.05$ \\
M/F & $25.99 \pm 3.455$ & $26.70 \pm 3.821$ & 0.14 \\
BMI, kg/m ${ }^{2}$ & $5(15.5 \%)$ & $22(11.1 \%)$ & 0.77 \\
Congestive heart failure, $\mathrm{n}(\%)$ & $3(9.3 \%)$ & $19(9.6 \%)$ & 0.91 \\
COPD, $\mathrm{n}(\%)$ & $21(65.6 \%)$ & $110(55.8 \%)$ & 0.32 \\
Hypertension, $\mathrm{n}(\%)$ & $14(43.7 \%)$ & $54(27.4 \%)$ & $<0.05$ \\
Diabetes, $\mathrm{n}(\%)$ & $2(6.2 \%)$ & $8(4.0 \%)$ & 0.68 \\
Chronic renal disease, $\mathrm{n}(\%)$ & $4(12.5 \%)$ & $9(4.5 \%)$ & $<0.05$ \\
Thyroid disease, $\mathrm{n}(\%)$ & $3(9.3 \%)$ & $8(4.0 \%)$ & 0.38 \\
Cerebrovascular disease, $\mathrm{n}(\%)$ & $2(6.2 \%)$ & $7(3.5 \%)$ & 0.47 \\
Carotid artery disease, $\mathrm{n}(\%)$ & $2(6.2 \%)$ & $4(2.0 \%)$ & 0.66 \\
Prophylactic IABP support, $\mathrm{n}(\%)$ & $2.514 \pm 1.687$ & $1.706 \pm 1.939$ & 0.021 \\
EurosCORE points & $1.188 \pm 0.396$ & $1.845 \pm 0.363$ & $<0.0001$ \\
Number of grafts, mean $\pm \mathrm{SD}$ & 0 & $98.28 \pm 35.09$ & $<0.001$ \\
CBP time, min & & & \\
\hline
\end{tabular}

BMI - body mass index, COPD - chronic obstructive pulmonary disease

group had an ICU stay of six months and died due to various complications that involved multiple organs and systems.

\section{DISCUSSIONS}

Being one of the most prevalent causes of death worldwide, there have been major developments regarding treatment strategies for coronary artery disease, in order to improve quality of life, related comorbidities, and survival. Although CABG is the routine and most frequently applied treatment of choice, accumulated evidence revealed various negative impacts on organ systems, particularly in high-risk individuals. Therefore, cardiovascular surgeons are in search of alternative strategies to

TABLE 2. Early postoperative outcomes between the two groups

\begin{tabular}{|c|c|c|c|}
\hline & $\begin{array}{c}\text { On-pump } \\
\text { beating-heart } \\
\text { surgery } \\
(\mathbf{n}=32)\end{array}$ & $\begin{array}{l}\text { Off-pump surgery } \\
(\mathbf{n}=197)\end{array}$ & $\mathrm{p}$ value \\
\hline In-hospital mortality, n (\%) & $1(3.12 \%)$ & $4(2.03 \%)$ & 0.78 \\
\hline Pulmonary complications, n (\%) & $1(3.12 \%)$ & $4(2.03 \%)$ & 0.78 \\
\hline Gastrointestinal complications, n (\%) & 0 & $1(0.5 \%)$ & 0.384 \\
\hline Renal failure requiring hemodialysis, $\mathrm{n}(\%)$ & 0 & $1(0.5 \%)$ & 0.384 \\
\hline Reoperation for bleeding, n (\%) & 0 & $5(2.53 \%)$ & 0.09 \\
\hline Arrythmia and atrial fibrillation, $\mathrm{n}(\%)$ & $4(12.5 \%)$ & $5(2.53 \%)$ & $<0.05$ \\
\hline Wound infection, n (\%) & 0 & $2(1.01 \%)$ & $<0.05$ \\
\hline Postoperative extubation duration, hours & $20.94 \pm 30.63$ & $12.08 \pm 12.01$ & 0.222 \\
\hline Length of ICU stay, days & $1.581 \pm 1.478$ & $1.219 \pm 0.8460$ & 0.098 \\
\hline Length of hospital stay, days & $10.97 \pm 4.254$ & $11.13 \pm 4.100$ & 0.717 \\
\hline Carotid artery disease, $\mathrm{n}(\%)$ & $2(6.2 \%)$ & $7(3.5 \%)$ & 0.47 \\
\hline Prophylactic IABP support, n (\%) & $2(6.2 \%)$ & $4(2.0 \%)$ & 0.66 \\
\hline EuroSCORE points & $2.514 \pm 1.687$ & $1.706 \pm 1.939$ & 0.021 \\
\hline Number of grafts, mean \pm SD & $1.188 \pm 0.3966$ & $1.845 \pm 0.3631$ & $<0.0001$ \\
\hline
\end{tabular}


overcome intraoperative and postoperative complications due to several interventions and manipulations during the course of CABG surgery.

$\mathrm{OBPH}-\mathrm{CABG}$ surgery is a relatively novel, hybrid method, preserving the heartbeat and blood flow during the operation, hence preventing myocardial damage and related adverse events. The complications related to aortic crossclamping or cardioplegic arrest during conventional CABG surgery have been well documented in patients with poor preoperative condition. ${ }^{9,11}$

In our study, we aimed to compare the operative and early postoperative data of two patient groups that underwent CABG with OBPH and off-pump surgery modalities. We found that there were no significant differences between the OBPH and off-pump surgery groups with regards to postoperative extubation duration, length of ICU and hospital stay, and complications. On the other hand, a higher EuroSCORE II in the OBPH surgery group suggested a higher risk profile for this group of patients, irrespective of age and cardiovascular, pulmonary, renal, and cerebrovascular comorbidities.

Our findings are consistent with the data of several research groups comparing the two operative modalities for similar variables. Recent studies have concluded that $\mathrm{OBPH}-\mathrm{CABG}$ surgery is equivalent or superior to off-pump surgery in different risk groups of patients. In their cohort of patients with a left ventricular ejection fraction (LVEF) of $<35 \%$, Xia et al. and Gamal et al. have reported that $\mathrm{OBPH}-\mathrm{CABG}$ had improved cardiac function in the early postoperative period, suggesting that this technique is an efficient alternative to off-pump surgery in patients with severely reduced LVEF. ${ }^{12,13}$ Although the former also concluded that the on-pump group had received a higher number of bypass grafts, our findings contradict their data. However, while their study included patients with at least 3 grafts, none of the patients in our cohort underwent CABG for more than 2 grafts. This variation might be due to the increased variability in patient selection criteria between surgical teams, and also to the experience of the surgical, antesthesiology, and postoperative reanimation teams.

Matkovic et al. conducted a long-term follow-up study comparing the outcomes of two different surgical teams. They concluded that on-pump and off-pump surgery did not differ significantly in terms of cardiovascular and cerebrovascular outcomes and long-term survival. They also reported that off-pump surgery was related to repeated hospitalizations for cardiac complaints during the five-year follow-up. ${ }^{1 / 4}$

The complications of CABG on cardiovascular, pulmonary, neurological, and renal systems have been docu- mented and reported worldwide. ${ }^{15-18}$ Although not having reached statistical significance, the ratios of postoperative complications related to the gastrointestinal and renal systems, reoperation for bleeding, and wound infection were higher in the off-pump group. This finding may be the consequence of adequate circulation support and preserved blood flow during OBPH surgery. Despite the suggestion that reoperation for bleeding might be related to additional heparinization, none of the patients in our study group with this complication received additional doses of heparin or drugs with a similar effect on platelets and the coagulation cascade. ${ }^{12}$

On the other hand, a relatively higher incidence of postoperative pulmonary complications in the on-pump group might be a result of extracorporeal circulation during surgery. However, the sole patient with complications in this group, a 74-year-old male with multiple chronic comorbidities, had died due to additional complications from delayed extubation on the 7th postoperative day.

Four patients that deceased during hospitalization had a poor clinical status with a EuroSCORE II $>6$, whereas one patient in the off-pump group, with a lower EuroSCORE II score and 189 minutes of CBP time, could not be extubated during the postoperative period and had deceased on the 7th postoperative day due to pulmonary complications.

The incidence of arrythmia and atrial fibrillation was significantly higher in the on-pump group, consistent with the findings of different studies with larger study populations, and also with the conclusions of meta-analyses. ${ }^{19,20}$ Although none of the patients had deceased due to complications resulting from arrythmia and atrial fibrillation in our study group, their deaths were related to an increased risk of hemodynamic instability and thromboembolic events. The reason for the superiority of offpump surgery in terms of arrythmia and atrial fibrillation is still under debate. Although atrial cannulation during on-pump surgery might be the main underlying cause, some suggest that the concomitant use of cardiopulmonary bypass may influence the postoperative new-onset arrythmia and atrial fibrillation. ${ }^{21}$

There are several limitations to our study. Firstly, this is a single-center, retrospective clinical chart data-collection study, with a small sample size and short followup duration, which may have influenced the comparative analyses between groups. Secondly, we did not subgroup the patients according to LVEF, and the study groups included patients with various degrees of impaired left ventricular function. Last but not least, although the complications were determined according to several clinical and paraclinical factors, including results obtained from blood 
specimens, we did not include data regarding serum levels of cardiovascular and acute renal injury markers in the present analysis.

\section{CONCLUSIONS}

In conclusion, on-pump beating-heart CABG surgery might be an efficient alternative to off-pump surgery with similar postoperative outcomes of in-hospital mortality or morbidity. Further studies that would include larger study populations, in high-volume centers, with inclusion of additional comorbidities, with longer follow-up duration are needed for a precise analysis of possible complications and fesability of this method.

\section{CONFLICT OF INTEREST}

Nothing to declare.

\section{REFERENCES}

1. Gaudino M, Angelini GD, Antoniades C, et al. Off-Pump Coronary Artery Bypass Grafting: 30 Years of Debate. J Am Heart Assoc. 2018;7:e009934. doi: 10.1161/JAHA.118.009934.

2. Malvindi PG, Margari V, Mastro F, et al. External aortic crossclamping and endoaortic balloon occlusion in minimally invasive mitral valve surgery. Ann Cardiothorac Surg. 2018;7:748-754. doi: 10.21037/acs.2018.10.09.

3. Moss E, Puskas JD, Thourani VH, et al. Avoiding aortic clamping during coronary artery bypass grafting reduces postoperative stroke. J Thorac Cardiovasc Surg. 2015;149:175180. doi: 10.1016/j.jtcvs.2014.09.011.

4. Al-Sarraf N, Thalib L, Hughes A, et al. Cross-clamp time is an independent predictor of mortality and morbidity in lowand high-risk cardiac patients. Int J Surg. 2011;9:104-109. doi: 10.1016/j.ijsu.2010.10.007.

5. Sarkar M, Prabhu V. Basics of cardiopulmonary bypass. Indian J Anaesth. 2017;61:760-767. doi: 10.4103/ija.IJA_379_17.

6. Wang W, Wang Y, Piao H, et al. Early and Medium Outcomes of On-Pump Beating-Heart versus Off-Pump CABG in Patients with Moderate Left Ventricular Dysfunction. Braz J Cardiovasc Surg. 2019;34:62-69. doi: 10.21470/1678-9741-2018-0207.

7. Hannan EL, Wu C, Smith CR, et al. Off-pump versus on-pump coronary artery bypass graft surgery: differences in shortterm outcomes and in long-term mortality and need for subsequent revascularization. Circulation. 2007;116:1145-1152. doi: 10.1161/CIRCULATIONAHA.106.675595.

8. Chikwe J, Lee T, Itagaki S, Adams DH, Egorova NN. LongTerm Outcomes After Off-Pump Versus On-Pump Coronary
Artery Bypass Grafting by Experienced Surgeons. J Am Coll Cardiol. 2018;72:1478-1486. doi: 10.1016/j.jacc.2018.07.029.

9. Bakaeen FG, Thourani VH. Is off-pump CABG off base? J Am Coll Cardiol. 2018;72:1487-1489. doi: 10.1016/j.jacc.2018.07.030.

10. Puskus JD, Martin J, Cheng DC, et al. ISMICS consensus conference and statements of randomized controlled trials of off-pump versus conventional coronary artery bypass surgery. Innovations (Phila). 2015;10:219-229. doi: 10.1097/ IMI.0000000000000184.

11. Gomes WJ, Angelini GD. On- or Off-pump Coronary Artery Bypass Surgery. Is the Debate Settling Down? Braz J Cardiovasc Surg. 2019;34:VIII-XI. doi: 10.21470/1678-9741-2019-0281.

12. Xia L, Ji Q Song $\mathrm{K}$, et al. Early clinical outcomes of onpump beating-heart versus off-pump technique for surgical revascularization in patients with severe left ventricular dysfunction: the experience of a single center. J Cardiothorac Surg. 2017;12:11. doi: 10.1186/s13019-017-0572-X.

13. Gamal A-S, Farag I. On-pump beating versus arrested heart (conventional) revascularization in severe left ventricular dysfunction. J Egypt Soc of Cardio-Thorac Surg. 2017;25:398404. doi: 10.1016/j.jescts.2017.05.007.

14. Matkovic M, Tutus V, Bilbija I, et al. Long Term Outcomes of The Off-Pump and On-Pump Coronary Artery Bypass Grafting In A High-Volume Center. Sci Rep. 2019;9:8567. doi: 10.1038/ s41598-019-45093-3.

15. McDonagh DL, Berger M, Mathew JP, Graffagnino C, Milano CA, Newman MF. Neurological complications of cardiac surgery. Lancet Neurol. 2014;13:490-502. doi: 10.1016/S14744422(14)70004-3.

16. Mali S, Haghaninejad H. Pulmonary complications following cardiac surgery. Arch Med Sci Atheroscler Dis. 2019;4:e280-e285. doi: 10.5114/amsad.2019.91432.

17. Pooria A, Pourya A, Gheini A. Postoperative complications associated with coronary artery bypass graft surgery and their therapeutic interventions. Future Cardiol. 2020;16:481-496. doi: 10.2217/fca-2019-0049.

18. Çakalağaoğlu KC, Selçuk E, Erdem H, Elibol A, Köksal C. Analysis of Readmissions to The Intensive Care Unit After Coronary Artery Bypass Surgery: Ten Years' Experience. Braz J Cardiovasc Surg. 2020;35:732-740. doi: 10.21470/1678-97412019-0299.

19. Ascione R, Caputo M, Calori G, Lloyd CT, Underwood MJ, Angelini GD. Predictors of atrial fibrillation after conventional and beating heart coronary surgery: a prospective, randomized study. Circulation. 2000;102:1530-1535. doi: 10.1161/01. cir.102.13.1530.

20. Järvinen O, Laurikka J, Tarkka MR. Off-pump versus on-pump coronary bypass. Comparison of patient characteristics and early outcomes. J Cardiovasc Surg (Torino). 2003;44:167-172.

21. Velioglu Y, Isik M. Early-Term Outcomes of Off-Pump versus On-Pump Beating-Heart Coronary Artery Bypass Surgery. Thorac Cardiovasc Surg. 2019;67:546-553. doi: 10.1055/s0039-1679923. 